Field-Capable Biodetection Devices for Homeland Security Missions

G. M. Dougherty, D. S. Clague, R. R. Miles

April 10, 2007

SPIE Defense and Security Symposium

Orlando, FL, United States

April 9, 2007 through April 13, 2007 
This document was prepared as an account of work sponsored by an agency of the United States Government. Neither the United States Government nor the University of California nor any of their employees, makes any warranty, express or implied, or assumes any legal liability or responsibility for the accuracy, completeness, or usefulness of any information, apparatus, product, or process disclosed, or represents that its use would not infringe privately owned rights. Reference herein to any specific commercial product, process, or service by trade name, trademark, manufacturer, or otherwise, does not necessarily constitute or imply its endorsement, recommendation, or favoring by the United States Government or the University of California. The views and opinions of authors expressed herein do not necessarily state or reflect those of the United States Government or the University of California, and shall not be used for advertising or product endorsement purposes. 


\title{
Field-Capable Biodetection Devices for Homeland Security Missions
}

\author{
George M. Dougherty, David S. Clague and Robin R. Miles \\ Lawrence Livermore National Laboratory, 7000 East Avenue, L-222, Livermore, CA 94551
}

\begin{abstract}
Biodetection instrumentation that is capable of functioning effectively outside the controlled laboratory environment is critical for the detection of health threats, and is a crucial technology for Health Security. Experience in bringing technologies from the basic research laboratory to integrated fieldable instruments suggests lessons for the engineering of these systems. This overview will cover several classes of such devices, with examples from systems developed for homeland security missions by Lawrence Livermore National Laboratory (LLNL). Recent trends suggest that front-end sample processing is becoming a critical performance-determining factor for many classes of fieldable biodetection devices. This paper introduces some results of a recent study that was undertaken to assess the requirements and potential technologies for next-generation integrated sample processing.
\end{abstract}

Keywords: Biodetection, biosensor, homeland security, autonomous, sample processing, sample preparation, requirements, diagnostics

\section{INTRODUCTION}

Over the past decade and more, there has been considerable investment in the development of devices and systems for the rapid detection and identification of biological agents. This investment has accelerated since the events of September and October 2001. While scientific laboratory techniques for microbiology and diagnostics have been advancing for some time, the last few years are uniquely marked by the rise of serious requirements for systems that can be deployed outside of the laboratory environment ${ }^{1-3}$. Primary application areas in homeland security include the unattended monitoring of ambient air for aerosolized biological agents, the rapid assessment and diagnosis of potentially infected humans or animals in the field, and the on-site assessment of potentially contaminated locations and the effectiveness of decontamination procedures. The development of field-capable biodetection devices has required the combination of biological assay technology with a range of other technologies in electronics, optics, mechanical design, automated fluidics, and system integration. The experience gained through the testing and deployment of such devices yields important guidance for the next generations of development efforts, including new technology development priorities.

Some of the application areas overlap with similar instrumentation needs for military applications. Two classes of systems have been primary subjects of engineering research and development effort: aerosol point detection systems, and field diagnostic and assessment systems. A brief survey of recent developments and the state of the art in these areas follows, including descriptions of representative systems developed at LLNL for homeland security applications.

\section{AEROSOL POINT DETECTION SYSTEMS}

Aerosol point detection systems are tasked with screening the air in the immediate ambient environment for biological agents. They can be divided into two main classes: detect-to-warn and detect-to-treat. Detect-to-warn devices detect the presence of a dangerous biological agent and provide an alarm in time for measures to be taken to limit or prevent the exposure of personnel. This is a demanding requirement, since for most situations a practical warning must come within a matter of 1-5 minutes ${ }^{1,3}$. Detect-to-treat devices aim to detect an agent in time for exposed personnel to be identified and receive prophylactic treatment, such as pre-symptomatic treatment with antibiotics. Because personnel will already 
be exposed by the time the detection can be made, it is usually necessary for the agent to be definitively identified as well, so that the appropriate mitigating action can be determined. It is possible to define three distinct generations of systems that have either been fielded or are currently in development.

\subsection{First-generation (attended) systems}

In the year 2001, the Department of Energy was completing a Domestic Demonstration and Application Program (DDAP) called Bioaerosol Sentry and Information System (BASIS), focused on the demonstration of a simple bioattack detection system for domestic security applications. Following the anthrax incidents of that autumn, the technologies demonstrated in this program were rapidly expanded to provide the basis for the primary, currently deployed civil biodetection program, called BioWatch. A similar Department of Defense program, called Guardian, was established to provide biodetection for military installations.

The first-generation systems consist of aerosol collectors, such as the BioWatch collector shown in Fig. 1, and regional diagnostic laboratories. The aerosol collectors do not contain any detection capability, but simply draw ambient air through a filter for a fixed period. On a daily schedule, the filter modules are replaced, and the exposed ones sent to a diagnostic laboratory for analysis using benchtop sample processing and assay techniques. Because of the time delay inherent in transferring the filters to the centralized laboratories, this is a detect-to-treat system, with delay times that can be longer than optimal to properly identify and treat the exposed individuals.

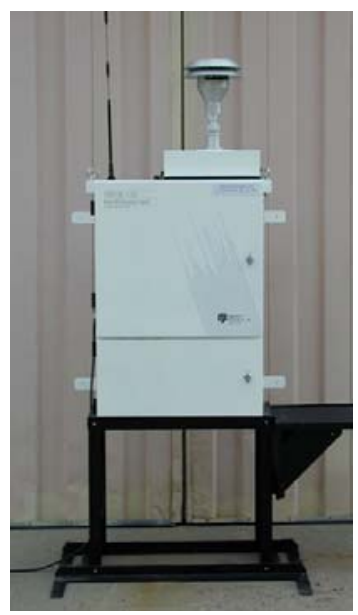

Fig. 1. One type of field aerosol collector used by the BioWatch system. Ambient air is drawn through the intake at the top of the unit, and passed through a filter to capture and retain any biological aerosol particles.

BioWatch is the current "gold standard" for bio-aerosol monitoring in homeland security roles. In addition to their ongoing operational importance, the first-generation systems play the role of establishing a performance baseline for subsequent developmental systems.

\subsection{Second generation (autonomous) systems}

In order to shorten the time between an attack and its detection, it is desirable to provide a point detector with its own capability to perform automated analyses of collected samples. Thus were developed a new generation of autonomous aerosol point detection systems. An example is the Autonomous Pathogen Detection System (APDS), developed at Lawrence Livermore National Laboratory with funding from the Department of Homeland Security (DHS), and shown in Fig. 2. Other examples include the Biohazard Detection System (BDS), used by the US Postal Service to detect anthrax contamination in mail processing facilities, and the Joint Biological Point Detection System (JBPDS) developed for military use. These systems are currently in advanced test and evaluation or have been deployed for field use.

For example, the APDS is an autonomous system designed for use in civil facilities such as subway stations, airports, and office buildings ${ }^{4-6}$. It contains an aerosol collector, including gas-phase particle filtration and a wetted-wall cyclone, 
serving to transfer the aerosol particles in the size range of biological agents into a volume of a few milliliters of water. Once concentrated into the liquid phase, the resulting sample is processed and analyzed using a bead-based multiplex immunoassay. A collection of antibody-coated fluorescent beads is maintained on board, each sensitive to a different biothreat agent. If the automated optical readout detects an apparent reaction between the sample and any of the selection of antibody-coated fluorescent beads, a confirmatory assay based on real-time polymerase chain reaction (PCR) nucleic acid detection is automatically conducted on another aliquot of the sample. The integration of this functionality into an autonomous sensor platform reduces the detection time to as little as 30-60 minutes, with specific identification of the agent. The combination of two independent assay types results in a very low rate of false alarms.

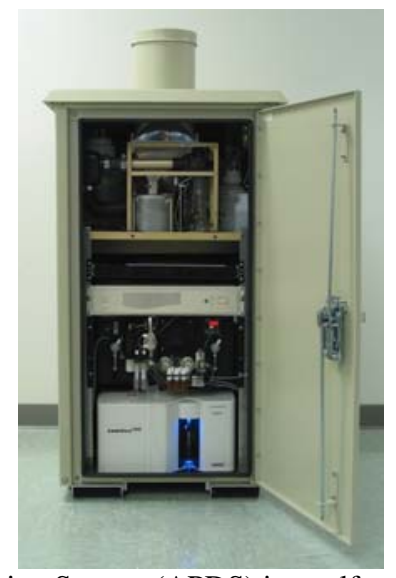

Fig. 2. The Autonomous Pathogen Detection System (APDS) is a self-contained biodetection system with automated sample collection, computer-controlled fluidics, wireless communications, and two independent types of bioassays.

The above second generation systems are detect-to-treat systems capable of identifying a detected biological agent. Concurrent with their development have been autonomous detect-to-warn point detection sensors that employ optical or other physical detection mechanisms to provide a rapid, but much less specific, warning of a possible attack. This is done by, for instance, detecting an increase in the UV fluorescence of the ambient air that might be indicative of a rise in the concentration of biological particles. In some cases, such as later versions of the JBPDS, such a detect-to-warn sensor is used as a trigger to initiate the collection and testing of a sample by an identification-capable host system.

\subsection{Next-generation systems}

Advanced development programs are currently pursuing autonomous detection systems that are smaller, provide even more rapid detection, and include extensive information networking capabilities. The DHS's Bioagent Autonomous Networked Detectors (BAND) and Rapid Automated Biological Identification System (RABIS) programs are funding the development of several concepts specifically targeted toward homeland security applications. An example is the BioBriefcase system under development at Lawrence Livermore National Laboratory and Sandia National Laboratory. A view of some of the key components of this system is shown in Fig. 3. Similar in basic operational architecture to the APDS, the BioBriefcase achieves a smaller form factor and shorter detection times by using a more highly integrated fluidic platform including some microfluidic components, and novel assays based on eTag ${ }^{\circledR}$ technology (ACLARA BioSciences) that allow for simpler and more compact assay readout mechanisms than the flow cytometer used in the APDS.

Concurrently, the Department of Defense is pursuing the development of sensors for military biodetection that may find use in homeland security scenarios as well. Because military environments offer low regret responses that may not be available in civilian environments, such as the donning of protective gear, military systems can be less specific and are therefore more focused on rapid detect-to-warn use. Like the systems under development by the DHS, these autonomous sensors are intended for long-term unattended use and integration with wireless data networks, enabling their use in emerging network-centric warfare architectures. 


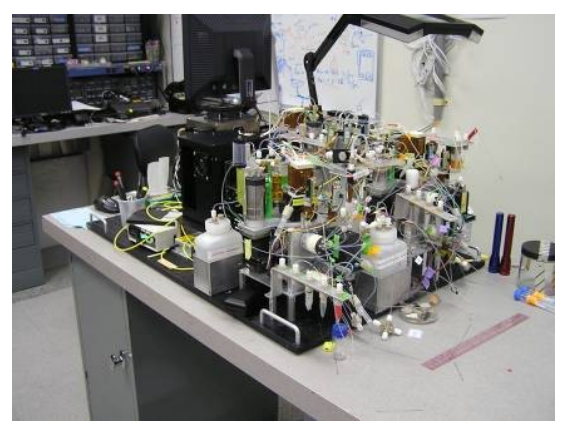

Fig. 3. A benchtop prototype of the BioBriefcase third-generation point detection system. Such systems contain miniaturized or microfluidic components, enhanced performance and extensive data networking capabilities.

\section{FIELD DIAGNOSTIC AND ASSESSMENT SYSTEMS}

Aerosol point detection sensors are not the only systems being developed for biodetection in the field. Other essential missions require a similarly high level of operational robustness and sample-to-answer automation. Rapid medical diagnostics and triage require the ability to perform complete biodetection tests on medical samples using compact systems that can operate reliably in field conditions. Similarly, first responders require the ability to perform on-site assessment of areas potentially contaminated with biological agents. When sites are confirmed to be contaminated and decontamination procedures are carried out, the personnel carrying out these operations need systems that can help them assess the effectiveness of the decontamination, and know when the job is complete. As with other biodetection mission areas, there is a good deal of overlap between technologies specialized for military use and those designed for homeland security needs.

\subsection{Field-portable site assessment systems}

The simplest and most common field-portable biodetection devices continue to be lateral-flow immunoassays, similar to those used in home pregnancy tests. Also called handheld assays or HHAs, these are highly portable and rugged, are small and light, and require no power. These assays are very effective in the rapid detection of dissolved proteins such as biotoxins, but when used for the detection of organisms, particularly in raw or complex samples, they can exhibit limitations in sensitivity and specificity. Some systems, such as the RAMP unit produced by Response Biomedical Corp., employ more advanced implementations of cartridge-based immunoassays to improve performance and ease of use.

A range of new systems using nucleic acid based assay technology have been developed by several agencies. One of the first such systems was the Advanced Nucleic Acid Analyzer (ANAA), developed at Lawrence Livermore National Laboratory in the late $1990 \mathrm{~s}^{7}$. With an assay based on real-time polymerase chain reaction (PCR) and automated fluorescence readout, the ANAA was packaged as a field-rugged unit and underwent successful military field trials in the Middle Eastern desert. Its key components were soon further developed for integration in a handheld unit called the Handheld Advanced Nucleic Acid Analyzer (HANAA). With four independent PCR reaction chambers, miniaturized optics and electronics, and battery power, the HANAA is capable of performing highly sensitive and specific nucleic acid sequence-based detection assays in the field ${ }^{8}$. The HANAA technology is commercially available in the form of the Bio-Seeq device manufactured by Smiths Detection. Both devices are shown in Fig. 4.

The key real-time PCR technology has been implemented in several other specialized biodetection devices, such as the ruggedized Joint Biological Agent Identification and Diagnostic System (JBAIDS I) developed for the U.S. military by Idaho Technology. While the above systems, and many similar devices designed for benchtop laboratory use, have made real-time PCR a standard biodetection technique, they typically require manual sample preparation prior to the introduction of the sample into the PCR reaction chamber. As discussed below, this defines one of the key frontiers of research and development for field-capable biodetection systems. 

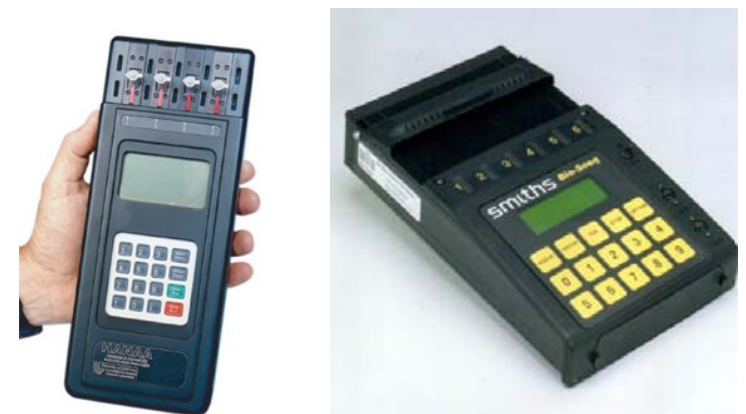

Fig. 4. The Handheld Advanced Nucleic Acid Analyzer (HANAA), at left, is an example of a field-portable biodetection system for use by first responders or decontamination crews. The core real-time PCR detection technology is also deployed in the commercial Bio-Seeq system (Smiths Detection), shown at right.

\subsection{Automated medical and veterinary diagnostics}

The missions for automated sample-to-answer medical diagnostic systems are broad, ranging from the rapid evaluation of potential human exposure to biological agents in the field and in front-line medical facilities, to on-site surveillance for animal-borne and zoonotic diseases in agricultural and veterinary settings. The biodetection mission is developing in parallel with the trend toward point-of-care diagnostics in the broader health care industry. To date, point-of-care instruments have focused on clinical chemistry such as blood gases and cardiac enzyme levels, but as the biodefensedriven technologies for rapid bioagent detection move forward, there is considerable synergy between the two fields.

It is likely that the first indication of a covert bioagent attack will be, as with a natural outbreak, the presentation of sick patients (or animals). Today, in up to $50 \%$ of serious respiratory infections the causative organism is never identified. In a bioattack response scenario, rapid identification of the agent is critical, and new systems are being developed to apply the multiplex rapid diagnostics developed for point detection application to this task. An example is the FluIDx system recently developed at Lawrence Livermore National Laboratory, a compact system based on technology from the APDS point detector. The simple, push-button system accepts a nasopharyngeal sample and automatically screens for 5 common upper respiratory viruses, a panel that can be easily expanded to additional pathogens. The FluIDx system is shown in Fig. 5. Similar point-of-care diagnostic technology has been demonstrated for the rapid screening of cattle and other farm animals for pandemic-capable diseases such as foot and mouth disease (FMD).

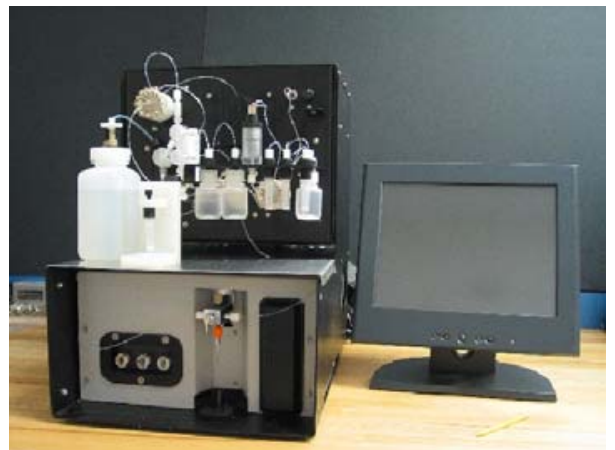

Fig. 5. The compact FluIDx unit is a point-of-care multiplex medical diagnostics system that uses the automated fluidics and bead-based immunoassay platform of the APDS. It has been used to rapidly diagnose viral upper respiratory infections.

\section{LESSONS FROM THE FIELD}

Many important lessons have been learned as a result of the development, field testing and deployment of biodetection instruments such as those described. These include issues such as power sources, data networking, logistical support, human factors engineering, and operational cost models. Perhaps the most pervasive technical theme to arise is sample 
processing. Regardless of application area, sample processing technology has emerged as a key limiting factor for the performance of integrated biodetection systems for field use.

Many bioassay mechanisms, such as sandwich immunoassays, PCR, and newer techniques, demonstrate excellent performance when assaying well-controlled samples in laboratory settings, but encounter a myriad of challenges in handling the uncontrolled and complex samples that are encountered in the field. As an illustration, Fig. 6 shows a liquid sample extracted from the aerosol collector of an APDS point detection system installed in an urban subway station. The sample is so heavily loaded with background particles, such as ferrous metal dust, that it is opaque. Similarly difficult samples result from the extraction of particles from carpet and other surfaces in contamination assessment scenarios, the collection of nasal secretions and other bodily fluids for medical diagnostics, and essentially all other field biodetection scenarios. System performance metrics, ranging from effective limits of detection to maintenance intervals, are largely determined by sample-processing related issues.

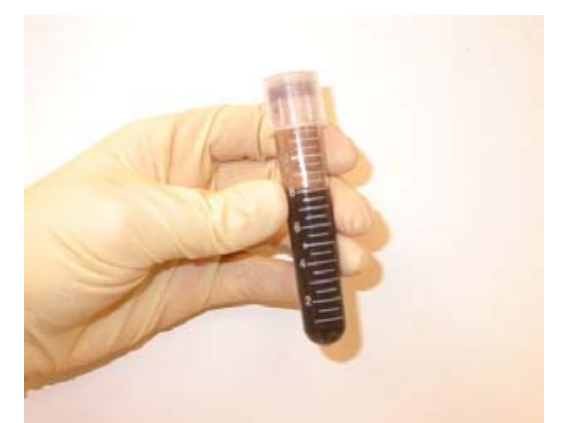

Fig. 6. A vial of liquid sample from an APDS system, showing the heavy background contamination and sample complexity encountered in field biodetection missions.

The ever tightening requirements for fast response, portability and ruggedness, assay multiplexing, low power consumption, high throughput and parallelism are driving a trend toward miniaturization. As mechanisms become smaller and more complex, there is an even greater need for effective sample processing to enable micro-engineered systems to handle these sorts of real-world samples.

\section{SAMPLE PROCESSING CHALLENGES}

In the modern diagnostic laboratory environment, bioassays as such occupy little space and account for a small fraction of the effort involved in a complete sample-to-answer analysis, the majority of space and time being consumed by the needs of sample processing, which constitutes the front portion of the sample-to-answer process. Centrifugation, filtration, lysis of spores, nucleic acid extraction, removal of inhibitory compounds, and other processes are often required before a raw sample is ready for final analysis ${ }^{9-13}$. In the context of fielded biodetection instruments, these processes can not be performed manually by skilled technicians, and have to be automated and integrated as functions of the instrument.

Some of the demands on integrated sample processing are inherent in the fact that fielded biodetection systems are compact and deployable, yet must exhibit high sensitivity. As first noted by Manz et al. ${ }^{14}$, and expanded by Peterson $^{15,16}$, the maximum sensitivity that can be achieved in a bioassay is limited by statistics. The smaller the working volume of an assay, the less sensitive it can be. In pathogen detection applications, critical concentrations can be as low as a few organisms per milliliter of sample. Since modern immunoassays and real-time PCR reactions have working volumes of $100 \mu \mathrm{L}$ or less and are getting smaller, efficient pre-concentration of the organisms is essential to achieve detection even under ideal conditions.

Similarly, the achievement of the expected detection performance often requires the removal of physically interfering contaminants as well as specifically inhibitory compounds. These differ depending on the operational scenario and the type of assay. 
The call for improved sample processing that results from field deployments of biodetection systems is seconded by the users and developers of laboratory-based bioanalytical systems, which are under pressure from the demands of highthroughput processing and the need to perform ever more, and higher performance, assays on smaller aliquots of clinical or environmental samples. With such consensus about the need for improved integrated sample processing capabilities, it may seem surprising that there has not been a corresponding focus on the development of these new capabilities. Sample processing has been, for the most part, a subordinate aspect of the development of assays and integrated systems, with limited priority placed on the innovation of new sample processing technologies as such. A key reason seems to have been the qualitative nature of the requirements. It has been difficult to come by requirements information that is sufficiently specific and quantitative to allow the assessment of technical options. The establishment of target metrics and their required values is essential, as is an ability to connect these sample processing subsystem metrics with more fundamental quantitative performance measures that can be levied on specific sample processing devices. A need has existed for the analytical background that can enable future sample processing technology programs to be established, and to guide researchers in developing new technologies responsive to the emerging system-level needs.

\section{SAMPLE PROCESSING STUDY}

To this end, Lawrence Livermore National Laboratory has undertaken a nine-month comprehensive study of sample processing for biodefense instrumentation, including both system-level requirements and the analysis of candidate physical mechanisms, as well as the specific technical constraints applied by various classes of downstream assays. The requirements analysis includes a review of the scholarly literature including existing technical studies and reports, surveys of the system-level state of the art, and an extensive series of interviews with 18 prominent biodefense-related agencies and institutions beyond LLNL. The sample processing mechanisms analysis covers 11 distinct approaches to separation, concentration, and extraction of particles and biochemical species. We believe this to be the most exhaustive study of the topic of integrated sample processing to date. Some preliminary findings of the study are summarized here.

\subsection{Mission areas}

Essential to the context of subsequent findings is that "biological sample processing" is not a single application area but consists of very distinct sets of requirements associated with a wide range of mission areas. The requirements study identified 10 major sample processing-relevant biodefense missions, each with distinct and generally non-overlapping requirements. The essential point is that there are no requirements for biological sample processing in the aggregate. System-level requirements and device development must consider the biodefense specific mission area and its particular needs and operational scenarios.

\subsection{Requirements Pull}

An important, if general, result of the information collected during the study is that it confirmed, and if possible, further established the widespread nature of the need for focused research effort in this area. A large fraction of the 19 total organizations cited sample processing as the most pressing technology need in their particular mission space, and essentially all of them listed it among their top priorities.

\subsection{Process breakdown}

The discussion of sample processing applications requires that distinctions be drawn between the various sub-processes that make up a complete sample processing sequence, from raw sample to treated analyte ready for output to the chosen assay. The requirements study yielded a consensus breakdown into sub-processes that can provide a canonical framework and vocabulary for these distinctions. The overall sequence is divided into sample pre-treatment, preextraction processing, extraction, and post-extraction processing, as illustrated in Fig. 7. Pre-treatment includes initial steps prior to introduction into the sample processing system proper, including aerosol collection, dilution in buffer, and other preliminary actions unique to each mission area. Pre-extraction processing refers to separation and/or concentration functions applied to particles and cells, prior to the extraction of biochemical analytes. Extraction is the processing of the resultant fraction to release the contained analytes of interest, and is of most importance in the 
processing of samples for nucleic acid based assays. Post-extraction processing includes the separation and/or concentration of dissolved species such as proteins and small molecules. Not all missions or assay types require all the steps in this canonical sequence, and sometimes two or more steps occur simultaneously as the result of a single process.

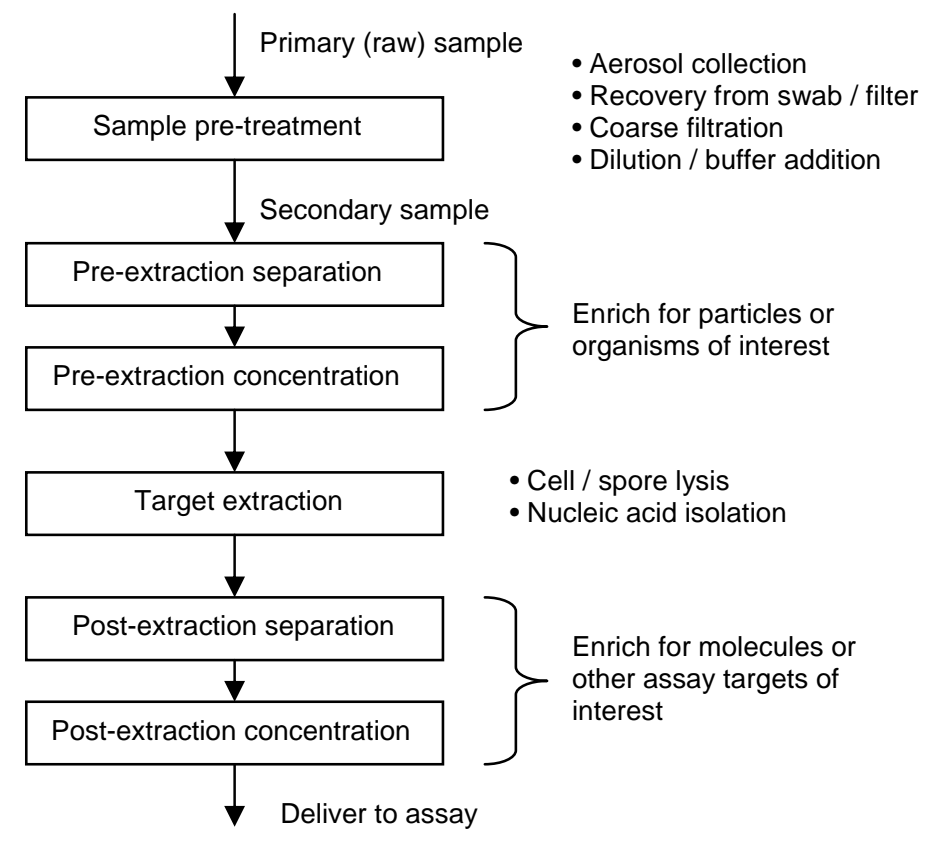

Fig. 7. Canonical process breakdown for a sample-to-analyte sample processing sequence. In practice, individual process steps may be omitted or combined.

\subsection{Parametric analyses}

The quantitative requirements data for the various biodefense mission areas can be usefully presented in the form of graphical parametric charts. These enable comparisons and contrasts, and illustrate relationships between the various mission area requirements that can reveal additional information. For example, in Fig. 8 below, requirements ranges for each of the 10 biodefense missions areas are plotted on two axes: sample-to-answer time and maximum throughput. The presentation of the information in this way indicates the relative needs for parallelism within each of the mission areas, i.e., the number of individual samples that must be processed simultaneously by a practical system.
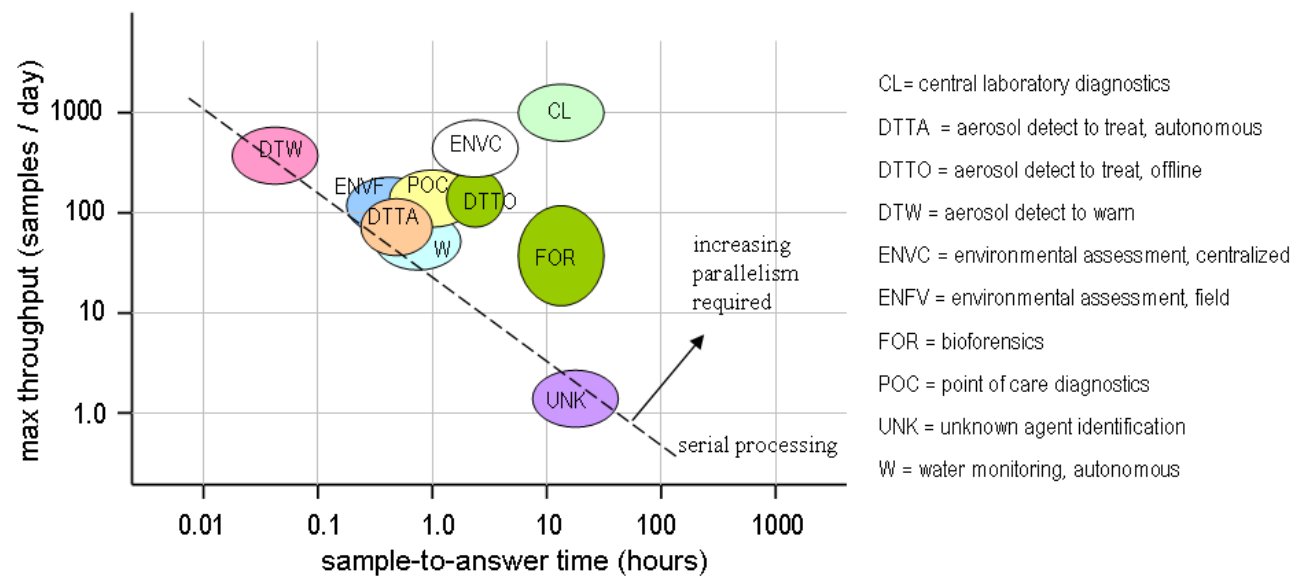

Fig. 8. An example of a parametric "bubble" chart showing the quantitative relationships between requirements for the 10 biodefense mission areas, with respect to detection speed and throughput. 
This extraction of values for system-level operational requirements parameters enables further digestion into requirements that can be levied on individual components within the system. Parametric analysis yields consensus ranges for such metrics as characteristic operating volumes, volumetric throughputs, and concentration ratios. These can be used to assess the feasibility of prospective technical approaches, and help ensure that research efforts to develop new component technologies target useful performance values.

\subsection{Non-technical requirements}

While much attention is focused on the technical requirements that must be met by the engineered sample processing systems, often the deciding factors for selection are non-technical in nature. These may be regulatory, or may reflect particular needs of the mission area. For instance, many point-of-care diagnostics applications require Clinical Laboratory Improvement Act (CLIA) waivability, and many counter-terrorism biodetection uses are subject to requirements on the legal admissibility and defensibility of assay results.

\section{CONCLUSION}

The realities of performing sensitive and reliable biodetection in the field add many requirements beyond those encountered in traditional laboratory-based testing. The growing needs for autonomous operation, long-term reliability with low maintenance, rapid sample-to-answer time, high sensitivity despite complex sample backgrounds, and similar qualities have been illustrated with examples from several key homeland security mission areas. Emerging trends, such as the micro-miniaturization of system components and the need to detect and identify unknown or modified biological agents, will place still greater pressure on such system-level requirements in the future. Experience has indicated that the most pervasive challenge in meeting these requirements may not be assay technology, but integrated sample processing. Focused research effort should be devoted to maturing the next generations of sample processing technologies. Some recent work is summarized that may help guide this effort, and ensure the advance of field-capable biodetection for homeland security, military, and health security needs.

\section{ACKNOWLDGEMENTS}

This work was performed under the auspices of the U. S. Department of Energy (DOE) by the University of California, Lawrence Livermore National Laboratory (LLNL) under Contract No. W-7405-Eng-48. The sample processing study was performed with funding from the Defense Advanced Research Projects Agency (DARPA), Strategic Technology Office.

\section{REFERENCES}

1. J. Vitko, ed., Sensor systems for biological agent attacks: protecting buildings and military bases, National Research Council, National Academies Press (2004).

2. J. Schultz, M. Mrksich, S. N. Bhatia, D. J. Brady, A. J. Ricco, D. R. Walt, and C. L. Wilkins, International research and development in biosensing, World Technology Evaluation Center (WTEC), August 2004.

3. J. F. O’Byron, ed., Defending the U.S. air transportation system against chemical and biological threats, National Research Council, National Academies Press (2006).

4. M. T. McBride, D. Masquelier, B. J. Hindson, A. J. Makarewicz, S. Brown, K. Burris, T. Metz, R. G. Langlois, K. W. Tsang, R. Bryan, D. A. Anderson, K. S. Venkateswaran, F. P. Milanovich, and B. W. Colston, Jr., "Autonomous detection of aerosolized Bacillus anthracis and Yersinia pestis,” Anal. Chem. 75(20), 5293-5299 (2003).

5. B. J. Hindson, A. J. Makarewicz, U. S. Setlur, B. D. Henderer, M. T. McBride, and J. M. Dzenitis, "APDS: the Autonomous Pathogen Detection System,” Biosensors and Bioelectronics 20, 1925-1931 (2005).

6. B. J. Hindson, M. T. McBride, A. J. Makarewicz, B. D. Henderer, U. S. Setlur, S. M. Smith, D. M. Gutierrez, T. R. Metz, S. L. Nasarabadi, K. S. Venkateswaran, S. W. Farrow, B. W. Colston, Jr., and J. M. Dzenitis, "Autonomous detection of aerosolized biological agents by multiplexed immunoassay with polymerase chain reaction confirmation,” Anal. Chem. 77(1), 284-289 (2005). 
7. P. Belgrader, W. Benett, D. Hadley, J. Richards, P. I. Stratton, R. Mariella, Jr. and F. Milanovich, "PCR analysis of bacteria in 7 minutes,” Science 284, 449-450 (1999).

8. P. A. Emanuel, R. Bell, J. D. Lang, R. Clanahan, J. C. David, R. J. Burgess, J. Thompson, L. Collins, and T. Hadfield, "Detection of Francisella tularensis within infected mouse tissues by using a hand-held PCR thermocycler,” J. Clin. Microbio. 41(2), 689-693 (2003).

9. R. Boom, C. J. Sol, M. M. Salimans, C. L. Jansen, P. M. Wertheim-van Dillen, J. van der Noordaa, "Rapid and simple method for purification of nucleic acids,” J. Clin. Microbio. 28(3) 495-503 (1990).

10. L. Lichtenberg, N. F. de Rooij, and E. Verpoorte, “Sample pretreatment on microfabricated devices,” Talanta 56, 233-266 (2002).

11. D. V. Lim, J. M. Simpson, E. A. Kearns and M. F. Kramer, “Current and developing technologies for monitoring agents of bioterrorism and biowarfare,” Clin. Microbio. Rev. 18(4) 583-607 (2005).

12. M. Toner and D. Irimia, “Blood-on-a-chip,” Annu. Rev. Biomed. Eng. 7, 77-103 (2005).

13. P. Belgrader, D. Hansford, G. T. A. Kovacs, K. Venkateswaran, R. Mariella, F. Milanovich, S. Nasarabadi, M. Okuzumi, F. Pourahmadi, and M. A. Northrup, “A minisonicator to rapidly disrupt bacterial spores for DNA analysis,” Anal. Chem. 71, 1999, 4232-4236.

14. A. Manz, N. Graber, and H. M. Widmer, "Miniaturized total chemical analysis systems: a novel concept for chemical sensing," Sensors and Actuators B 1, 244-248 (1990).

15. K. E. Petersen, W. A. McMillan, G. T. A. Kovacs, M. A. Northrup, L. A. Christel, and F. Pourahmadi, “Toward next generation clinical diagnostic instruments: scaling and new processing paradigms,” Biomed. Microdev. 1(1) 71-79 (1998).

16. K. E. Petersen, “The future of DNA diagnostic testing,” in Microfluidics and BioMEMS, SPIE vol. 4560, 1-7 (2001). 